The system of groups under consideration is of special interest because it includes $p-2$ non-abelian groups in which every operator except identity is of order $p$, for every possible value of the prime number $p$. The number of these groups for a particular prime number therefore depends upon this prime. The fact that the group generated by $s_{1}, s_{2}$ is completely determined by its order seems also worth noting. UNIVERSITY OF ILUINOIS, December, 1916.

\title{
A THEOREM IN THE ANALYSIS OF REAL VARIABLES.
}

BY PROFESSOR R. L. BORGER.

(Read before the American Mathematical Society, April 21, 1916.)

In this paper the following theorem is proved:

Theorem: If the two real functions $U(x, y), V(x, y)$ of the real variables $x, y$ satisfy the following conditions at each point of a closed region $R$ :

(a) $U$ and $V$ continuous in $x$ and $y$ jointly;

$$
\frac{\partial U}{\partial x} \equiv U_{1}, \quad \frac{\partial U}{\partial y} \equiv U_{2}, \quad \frac{\partial V}{\partial x} \equiv V_{1}, \quad \frac{\partial V}{\partial y} \equiv V_{2}
$$

exist and are finite;

(c) $\Delta U=h U_{1}+k U_{2}+\rho_{1}(h, k), \quad \Delta V=h V_{1}+k V_{2}+\rho_{2}(h, k)$;

(d) $\operatorname{Lim}_{h, k \doteq 0} \frac{\left|\rho_{i}(h, k)\right|}{|h|+|k|}=0 \quad(i=1,2)$;

(e) $U_{1}=V_{2}, \quad U_{2}=-V_{1}$

then $U$ and $V$ are analytic functions of $x, y$ in $R$.

An immediate consequence of the theorem is that if any function $W$ of a complex variable $z$ possesses a finite derivative at each point of a simply connected closed region $R$ then:

1. This derivative is continuous.

2. All the derivatives of $W$ exist.

3. The function $W$ may be represented by a power series in $z$. 
To prove the theorem we apply the following, due to Kowalewski:*

If the two real functions $U(x, y), V(x, y)$ are properly differentiable (satisfy hypotheses $c, d$ ) and if

$$
U_{1}=V_{2}
$$

at each point of a rectangle $R$, there exists a function $\bar{U}(x, y)$ such that

$$
\bar{U}_{1}=U ; \quad \bar{U}_{2}=V .
$$

The function $U$ is, moreover, properly differentiable.

Employing this theorem we may infer the existence of two functions (in view of both parts of hypotheses $(e)) \bar{U}, \bar{V}$ such that

$$
\bar{U}_{1}=U, \quad \bar{U}_{2}=-V, \quad \bar{V}_{1}=V, \quad \bar{V}_{2}=U .
$$

From equations (1)

$$
\bar{U}_{1}=\bar{V}_{2} ; \quad \bar{U}_{2}=-\bar{V}_{1} .
$$

Since $\bar{U}$ and $\bar{V}$ are properly differentiable we have by a second application of Kowalewski's theorem, two other functions, $\bar{U}, \bar{V}$, also properly differentiable and such that

$$
\bar{U}_{1}=\bar{U} ; \quad \bar{U}_{2}=-\bar{V} ; \quad \bar{V}_{1}=\bar{V} ; \quad \bar{V}_{2}=\bar{U} .
$$

The functions $\overline{\bar{U}}(x, y), \overline{\bar{V}}(x, y)$, as well as their first and second partial derivatives, are easily seen to be continuous in the variables $x, y$ and satisfy Laplace's differential equation $\Delta U=0$, since

$$
\begin{gathered}
\frac{\partial^{2} \overline{\bar{U}}}{\partial x^{2}}=\frac{\partial \bar{U}_{1}}{\partial x}=\frac{\partial \bar{U}}{\partial x}=\bar{U}_{1}=U, \\
\frac{\partial^{2} \bar{U}}{\partial y^{2}}=\frac{\partial \bar{U}_{2}}{\partial y}=-\frac{\partial \bar{V}}{\partial y}=-V_{2}=-U . \\
\therefore \frac{\partial^{2} \bar{U}}{\partial x^{2}}+\frac{\partial^{2} \bar{U}}{\partial y^{2}}=0 .
\end{gathered}
$$

Similarly for $\overline{\bar{V}}$.

The functions $\bar{U}, \bar{V}$ are then analytic $\dagger$ functions of $x, y$.

* Kowalewski, Die komplexen Veränderlichen und ihre Funktionen, p. 187.

$\dagger$ Picard, Traité d'Analyse, tome 2 (2d ed.), p. 18. 
The functions $U$ and $V$ being contained among the derivatives of $\bar{U}$ and $\bar{V}$ are also analytic functions of $x, y$.

It may now be seen that we have the following representation for these functions:

$$
\begin{gathered}
U(x, y)=\sum_{k=0}^{\infty}\left(a_{10} x+a_{01} y\right)^{k} ; \\
V(x, y)=\sum_{k=0}^{\infty}\left(b_{10} x+b_{01} y\right)^{k} ; \\
\left(\left(a_{10}\right)^{l}\left(a_{01}\right)^{m}=a_{l m} ; \quad\left(b_{10}\right)^{l}\left(b_{01}\right)^{m}=b_{l m} ; \quad l, m=0, \cdots, k\right) .
\end{gathered}
$$

Differentiating the equations

$$
U_{1}=V_{2}, \quad U_{2}=-V_{1}
$$

$l-1$ times with respect to $x$ and $k-l$ times with respect to $y$, at the point $(0,0)$ we have the following relations among the coefficients:

$$
a_{l, k-l}=b_{l-1, k-l+1} ; \quad b_{l, k-l}=-a_{l-1, k-l+1} \quad(l=1, \cdots, k) .
$$

We may now prove that if a function $W$ of a complex variable $z$ possesses a finite derivative at each point of a simply connected closed region, $R$,

1. This derivative is continuous.

2. All the derivatives of $W$ exist.

3. The function $W$ may be represented as a power series in $z$.

Since $U$ and $V$ are analytic functions of $x$ and $y$, we see immediately from the representation

$$
W=U(x, y)+i V(x, y)
$$

that 1 and 2 are true. From equations (6)

$$
\begin{aligned}
W & =U(x, y)+i V(x, y)=\sum_{k=0}^{\infty}\left(a_{10} x+a_{01} y\right)^{k}+i \sum_{k=0}^{\infty}\left(b_{10} x+b_{01} y\right)^{k} \\
& =\sum_{k=0}^{\infty} \sum_{l=0}^{k}\left(\begin{array}{l}
k \\
l
\end{array}\right)\left[a_{l, k-l}+i b_{l, k-l]}\right] x^{l} \cdot y^{k-l} .
\end{aligned}
$$

From equations (7) we have

$$
a_{l-1, k-l+1}+i b_{l-1, k-l+1}=i\left(a_{l, k-l}+i b_{l, k-l}\right) \quad(l=1, \cdots, k)
$$

and in general

$a_{l-j, k-l+j}+i b_{l-j, k-l+j}=(i)^{j}\left(a_{l, k-l}+i b_{l, k-l}\right) \quad(j=1, \cdots, l)$. 
For $l=k$ it follows that

Then

$$
a_{k-j, j}+i b_{k-j, j}=(i)^{j}\left(a_{k 0}+i b_{k 0}\right) \quad(j=1, \cdots, k) .
$$

$$
\begin{aligned}
W(z) & =\sum_{k=0}^{\infty}\left(a_{k 0}+i b_{k 0}\right)(x+i y)^{k} \\
& =\sum_{k=0}^{\infty} C_{k} z^{k}, \text { where }\left(C_{k} \equiv a_{k 0}+i b_{k 0}\right) .
\end{aligned}
$$

This completes the theorem.

I am indebted to Professor E. J. Townsend for suggesting the problem.

OHIO UNIVERSITY,

Athens, OHIo.

\section{CONCERNING THE COMPLEMENT OF A COUNT- ABLE INFINITY OF POINT SETS OF A CERTAIN TYPE.}

BY DR. J. R. KLINE.

(Read before the American Mathematical Society, December 27, 1916.)

IN his "Grundzüge der Mengenlehre," Hausdorff proved that if $E$ denotes a euclidean space of two or more dimensions while $R$ is a countable set of points belonging to $E$, then $E-R$ is a connected* point set. $\dagger$ It is the object of the present paper to prove a theorem, which contains Hausdorff's theorem as a special case. Hausdorff's method of proof does not apply for the proof of the more general theorem. While the proof is carried out for the case of two dimensions, it is evident that a similar proof would apply to any higher number of dimensions.

Theorem. If $M$ is a domain $\ddagger$ and $G_{1}, G_{2}, G_{3}, \ldots$ is a countable infinity of nowhere dense§ closed point sets, no one of

* A set of points is said to be connected if, however it be divided into two mutually exclusive proper subsets, one of them contains a limit point of the other.

$\dagger$ Cf. F. Hausdorff, "Grundzüge der Mengenlehre," Leipzig, Veit, 1914, p. 333.

$\ddagger \mathrm{A}$ domain is a connected set of points $M$, such that if $P$ is a point of $M$, then there exists a region containing $P$ and lying in $M$.

$\$$ A set of points is said to be nowhere dense, if in every region $R_{1}$ there exists a region $R_{2}$ entirely free of points of the set. A set is said to be closed if it contains all its limit points. 